\title{
Fbxw8 protein promotes the proliferation and metastasis of ESCC
}

\author{
Shanshan $\mathrm{Gao}^{1, *}$, Dongdong Zhao ${ }^{2}$ \\ ${ }^{1}$ Bethune international peace hospital, China \\ ${ }^{2}$ Meizhou people's Hospital, China
}

\begin{abstract}
Objective: This article plans to investigate Fbxw8 protein expression in esophageal squamous cell carcinoma tissues. And its effect on the proliferation ability of esophageal squamous cancer KYSE70 cells is analyzed. Methods: We can detect Fbxw8 protein expression in esophageal squamous cell carcinoma tissues with IHC and analysis the expression with the sex, age, lymph node metastasis and tumor stage. We alsodetect the proliferation of KYSE70 cells with CCK 8. Result: Most esophageal squamous carcinoma tissues highly expressed Fbxw8 protein, and the expression was positively correlated with lymph node metastasis and tumor staging $(\mathrm{P}<0.05)$. Fbxw8 siRNAs can significantly cut FBXW8 protein expression, while lowering the cell cycle related protein expression of Cyclin A, Cyclin B1, and inhibiting KYSE70 cell proliferation. Conclusion: Fbxw8 protein highly expressed in esophageal squamous carcinoma tissues, and closely related to tumor staging. Targeted inhibition Fbxw8 protein expression could significantly inhibit the proliferation of esophageal squamous carcinoma cells.
\end{abstract}

\section{Introduction}

Background: esophageal cancer is one of the most common malignant gastrointestinal tumors. The incidence rate of China's esophageal cancer is eighth [1] in all cancers worldwide. The incidence rate of esophageal cancer, especially esophageal squamous cell carcinoma, is significantly higher than that of [2] in western countries. The 5 year survival rate of advanced esophageal squamous cell carcinoma is about $10 \%[3]$. At present, radiotherapy and chemotherapy are still the main treatment methods for locally advanced or metastatic esophageal squamous cell carcinoma. However, more than $40 \%$ of patients died of tumor recurrence and metastasis, chemotherapy drug toxicity and immune diseases caused by radiotherapy and chemotherapy [4]. At present, the research on esophageal cancer is still far from enough. Many scholars at home and abroad are searching for the molecular level regulatory pathway and the target of treatment, in order to make a breakthrough in the treatment of esophageal squamous cell carcinoma.

Ubiquitin proteasome system is a selective degradation of intracellular proteins, which plays an important role in the process of tumor cell proliferation and metastasis [5]. It consists of: ubiquitin activating enzyme (E1), ubiquitin binding enzyme (E2) and ubiquitin ligase (E3) [6]. The F-box protein in ubiquitin ligase $\mathrm{E} 3$ is responsible for identifying the substrate to be degraded. Fbxw8 combines with cul1, cul7, Skp1 and roc1 in the Cullin family to form E3 ligase complex. Some studies have shown that fbxw 8 protein promotes the proliferation of tumor cells in colon cancer cells, prostate cancer cells and choriocarcinoma cells by regulating the cyclin of cells.

In this study, the expression of fbxw8 protein in esophageal squamous cell carcinoma and kyse 70 cell line were observed, and the effect of fbxw 8 protein on the proliferation of tumor cells was observed by targeting inhibition of fbxw8 protein expression, so as to provide a new target for the treatment of esophageal squamous cell carcinoma.

\section{Materials and methods}

\subsection{Information and main reagents}

From January 2016 to December 2016, 82 specimens of esophageal squamous cell carcinoma were collected from the Department of Pathology, the First Affiliated Hospital of Zhengzhou University. There were 55 males and 27 females. The age distribution ranged from 38 to 76 years, with a median age of 57 years. There were 10 cases with T stage T1, 29 cases with T2, 38 cases with T3 and 5 cases with T4. There were 31 cases without lymph node metastasis and 51 cases with lymph node metastasis AJCC [7] staging standard of China Cancer Joint Committee: 40 cases in stage I - II, 42 cases in stage III IV. All patients with esophageal squamous cell carcinoma were newly diagnosed.SP kit and DAB kit are from Beijing Bioson Biotechnology Co., Ltd.; human esophageal squamous cell carcinoma cell line kyse 70 is from ATCC. 1640 culture medium and fetal bovine

\footnotetext{
*Corresponding author: 13383614157@189.cn
} 
serum were purchased from hyclone company in the United States; pancreatin was the product of GIBCO company in the United States; all primers in this study were synthesized by Shanghai Biotechnology Co., Ltd.; Trizol was the product of Invitrogen company; cell counting Kit- 8 is a product of Tongren Chemical Research Institute of Japan Co., Ltd.; Rabbit anti human fbxw8 antibody comes from abgent company in the United States; polyclonal antibody of cyclin A, cyclin B1, GAPDH and Goat anti rabbit IgG polyclonal antibody labeled with horseradish peroxidase (HRP) are provided by doctoral Bioengineering Co., Ltd. in Wuhan.

\subsection{Method}

\subsubsection{The expression}

The expression of fbxw8 protein in esophageal carcinoma was detected by immunohistochemistry. After slicing the paraffin of esophageal cancer tissue samples, dewaxing and hydration were carried out; after that, the slides were placed in a $0.01 \mathrm{~mol} / \mathrm{L}$ buffer solution made of sodium citrate for microwave thermal antigen repair; at room temperature, $3 \% \mathrm{H} 2 \mathrm{O} 2$ was added for $30 \%$ reaction Min was used to eliminate endogenous peroxidase; at room temperature, goat serum was dripped to block for $30 \mathrm{~min} ; 1: 50 \mathrm{fbxw} 8$ antibody was dripped to block overnight at $4{ }^{\circ} \mathrm{C}$; the second day, the second antibody was dripped to react at room temperature for 30min; DAB color was developed, hematoxylin stained the nucleus; after dehydration, neutral gum was used to block, and then the staining results of slides were observed under microscope. Immunohistochemical scoring standard: each slide was randomly observed under 5-10 high power mirrors $(\times 400)$, and at least 100 cells could be counted in each field; then the immunohistochemical results were comprehensively judged according to the staining intensity (nucleus or cytoplasmic particles) and the percentage of positive cells. According to the staining intensity score: 0 for cell-free staining; 1 for pale yellow granules or masses; 2

table. the higher expression level of fbxw8 protein

\begin{tabular}{|c|c|c|c|c|}
\hline \multirow{2}{*}{ project } & \multicolumn{2}{|c|}{ Fbxw8 protein expression } & \multirow[b]{2}{*}{$\chi^{2}$} & \multirow[b]{2}{*}{$\mathrm{P}$} \\
\hline & High expression & low expression & & \\
\hline \multirow[t]{2}{*}{$\begin{array}{c}\text { No lymph node } \\
\text { metastasis }\end{array}$} & 21 & 10 & \multirow{3}{*}{4.030} & \multirow{3}{*}{0.045} \\
\hline & & & & \\
\hline Lymph node metastasis & 44 & 7 & & \\
\hline Phase I + II & 28 & 12 & \multirow{2}{*}{4.082} & \multirow{2}{*}{0.043} \\
\hline Phase III + IV & 37 & 5 & & \\
\hline$\geqslant 57$ years & 40 & 8 & \multirow{2}{*}{1.164} & \multirow{2}{*}{0.281} \\
\hline$<57$ years & 25 & 9 & & \\
\hline Male & 48 & 12 & \multirow{2}{*}{0.073} & \multirow[b]{2}{*}{0.787} \\
\hline Female sex & 17 & 5 & & \\
\hline
\end{tabular}

\subsection{The effect of fbxw8 protein}

The effect of fbxw8 protein on the proliferation of kyse70 cells. The stable growth tumor cells were transfected with negative control siRNA (CTL siRNA) for brownish yellow granules or masses; 3 for brownish brown granules or masses; according to the percentage of positive cells, 0 for positive cells $\leq 10 \%$; 1 for positive cells $10 \% \sim 50 \% ; 2$ for positive cells $>50 \% \sim 75 \% ; 3$ for positive cells The percentage is more than $75 \%$; then the above two scores are multiplied to get a comprehensive score: 0 points are $(-), 1-2$ points are $(+), 3-4$ points are $(++)$, 5-9 points are $(+++)$, among which, $\geq++$ is high expression and the rest is low expression.

Invitrogen designed fbxw8 steadthtm siRNA sequence: fbxw8siRNA:5 '- auuaccgucuguaaggcuugg-3. SiRNA sequence in Shanghai shenggonghe cost experiment.

\subsubsection{CCK-8}

CCK-8 test kit was used to measure cell proliferation. Kyse70 cells in logarithmic growth stage were inoculated in 96 well plates at a density of $1 \times 103 / \mathrm{ml}$ and cultured in $37{ }^{\circ} \mathrm{C}, 5 \% \mathrm{CO} 2$ incubator for 24 hours. They were transiently transfected with negative control siRNA (CTL siRNA) and fbxw8 siRNA for 24 hours, 48 hours, 72 hours and 96 hours, respectively. After that, 10ul CCK-8 hours. After that, the enzyme marker was used for 450 hours The absorbance of each hole was measured under nm light wave.

\section{Results}

The results of immunohistochemistry showed that the expression of fbxw8 protein was mainly located in the area around the cytoplasm and nucleus. The high expression rate of fbxw8 protein was 79.3\% (65/82). There was no significant correlation between the high expression rate and the age and gender of patients $(\mathrm{P}=$ $0.390, \mathrm{P}=0.348)$, which was closely related to lymph node metastasis and tumor stage $(\mathrm{P}<0.05)$. The more lymph node metastasis, the later stage, the higher expression level of fbxw8 protein, see table 1 . solution was added into each well and cultured for 2

and fbxw 8 siRNA respectively, and the expression of fbxw8 protein was verified by Western blot after culture in the incubator. In addition, the tumor cells transfected with CTL siRNA and fbxw8 siRNA were cultured in the incubator, and the proliferation capacity of the cells was 
measured by CCK- 8 test kit. Od $450 \mathrm{~nm}$ values of the two groups of tumor cells were measured four times at $24 \mathrm{~h}$, $48 \mathrm{~h}, 72 \mathrm{~h}$ and $96 \mathrm{~h}$, respectively. The results showed that compared with the CTL siRNA transfected cells, the proliferation ability of fbxw8 siRNA transfected cells decreased significantly, with the most significant decline at $72 \mathrm{~h}(\mathrm{P}=0.014$, figure 1$)$.

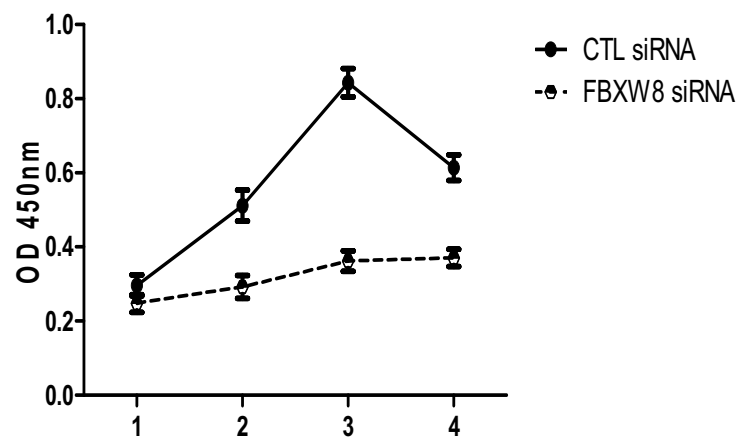

Fig.1. The proliferation ability of fbxw8 siRNA transfected cells decreased significantly

The effect of fbxw8 on the expression of cyclin in kyse70 cells. Western blot was used to detect the expression of cyclin in $\mathrm{G} 2$ / $\mathrm{M}$ phase. The results showed that the expression of Cyclina and cyclinB1 protein decreased in fbxw8 siRNA transfected cells compared with CTL siRNA transfected cells $(\mathrm{P}<0.05$, figure 2$)$.

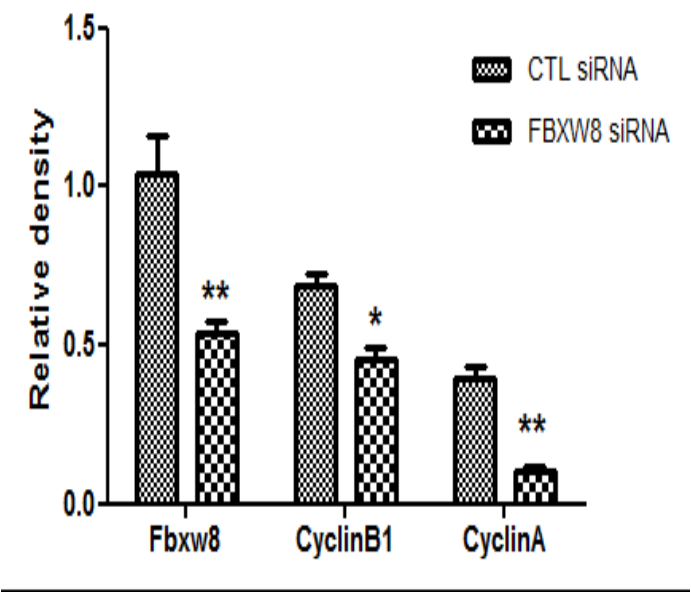

Fig.2. Fbxw8 siRNA transfected cells compared with CTL siRNA

\section{4 conclusion}

In this study, we found that fbxw8 protein was expressed in esophageal squamous cell carcinoma, and the high expression rate of fbxw8 protein was higher in patients with lymph node metastasis and AJCC stage III + IV. These results suggest that fbxw8 is closely related to the occurrence, development and metastasis of esophageal squamous cell carcinoma to a large extent. Fbxw8 protein may be one of the markers of the progression, invasion and metastasis of esophageal squamous cell carcinoma. To further understand the relationship between fbxw 8 protein and esophageal squamous cell carcinoma. By targeting fbxw8 siRNA to inhibit the expression of fbxw8 protein, we found that inhibition of fbxw8 protein significantly decreased the proliferation of kyse 70 cells, accompanied by the decreased expression of cyclin A and cyclin B1. It is suggested that the down-regulation of fbxw8 can increase the inhibitory factors of cyclin A and cyclin B1, arrest the division of kyse 70 cells, and inhibit the proliferation of cells.

\section{References}

1. Ekman S, Dreilich M, Lennartsson J, et al. Esophageal cancer: current and emerging therapy modalities[J]. Expert Rev Anticancer Ther, 2008, 8(9): 1433-48.

2. Siegel R, Naishadham D, Jemal A, et al. Cancer statistics, 2012[J]. CA Cancer J Clin, 2012, 62(1): 10-29.

3. Tachimori Y, Ozawa S, Numasaki $H$, et al. Supraclavicular node metastasis from thoracic esophageal carcinoma: A surgical series from a Japanese multi-institutional nationwide registry of esophageal cancer[J]. J Thorac Cardiovasc Surg, 2014, 148(4): 1224-9.

4. Aminian A, Panahi N, Mirsharifi R, et al. Predictors and outcome of cervical anastomotic leakage after esophageal cancer surgery[J]. J Cancer Res Ther, 2011, 7(4): 448-53.

5. $\mathrm{Xu} \mathrm{x,} \mathrm{Sarikas} \mathrm{A,} \mathrm{Dias-Santagata} \mathrm{DC,} \mathrm{et} \mathrm{a1.} \mathrm{The}$ CUL7 F3 ubi-quitin ligase targets insulin receptor substrate 1 for ubiquitin-de-pendent degradation[J]. Mol Cell, 2008, 30(4): 403-414.

6. Jin BF, He K, Wang HX, et a1. Proteomic analysis of ubiquitinproteasome effects: insight into the function of eukaryotic initiation factor $5 \mathrm{~A}[\mathrm{~J}]$. Oneogene, 2003, 22(31): 4819-4830.

7. Tsunematsu R, Nishiyama M, Kotoshiba S, et a1. Fbxw8 is essential for Cull-Cul7 complex formation and for placental development [J]. Mol Cell Biol, 2006, 26(16): 6157-6169.

8. Lin P, Fu J, Zhao B, et a1. Fbxw8 is involved in the proliferation of human choriocarcinoma JEG-3 cells[J]. Mol Biol Rep, 2011, 38(3): 1741-1747. 